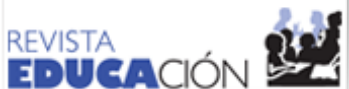

Revista Educación

ISSN: 0379-7082

ISSN: 2215-2644

revedu@gmail.com

Universidad de Costa Rica

Costa Rica

\section{La investigación universitaria sobre el blended learning en Perú: campos de conocimiento y tendencias metodológicas}

Turpo-Gebera, Osbaldo; Gonzales-Miñán, Milagros; García-Peñalvo, Francisco; Pari-Tito, Fernando

La investigación universitaria sobre el blended learning en Perú: campos de conocimiento y tendencias metodológicas

Revista Educación, vol. 44, núm. 2, 2020

Universidad de Costa Rica, Costa Rica

Disponible en: http://www.redalyc.org/articulo.oa?id=44062184037

DOI: https://doi.org/10.15517/revedu.v44i2.40743

Esta obra está bajo una Licencia Creative Commons Atribución-NoComercial-SinDerivar 3.0 Internacional. 


\section{La investigación universitaria sobre el blended learning en Perú: campos de conocimiento y tendencias metodológicas}

\section{University Research on Blended Learning in Peru: Fields of Knowledge and Methodological Trends}

Osbaldo Turpo-Gebera

Universidad de San Martin, Perú

oturpog@usmp.pe

(iD http://orcid.org/0000-0003-2199-561X

Milagros Gonzales-Miñán

Universidad de San Martin de Porres, Perú

mgonzalesm1@usmp.pe

ID http://orcid.org/0000-0003-2529-0174

Francisco García-Peñalvo

Universidad de Salamanca, España

fgarcia@usal.es

(iD) http://orcid.org/0000-0001-9987-5584

Fernando Pari-Tito

Universidad Nacional de San Agustín, Perú

fpari@unsa.edu.pe

(iD http://orcid.org/0000-0002-1429-544X
DOI: https://doi.org/10.15517/revedu.v44i2.40743

Redalyc: http://www.redalyc.org/articulo.oa?id=44062184037

Recepción: 26 Marzo 2020

Aprobación: 21 Julio 2020

\section{Resumen:}

El aprendizaje semipresencial (blended learning), como modalidad normalizada, presupone nuevas vinculaciones con los espacios y sujetos educativos, tornándose en objeto de investigación y conocimiento. El presente estudio recupera del Registro Nacional de Trabajos de Investigación (RENATI) 45 informes de tesis de pregrado y posgrado sustentados en las universidades peruanas. Con los informes se establecieron los campos de conocimiento y orientaciones metodológicas predominantes. Los resultados revelan una mayor concentración investigativa de estudios a nivel de postgrado, una creciente evolución productiva en el último quinquenio, así como intereses de desarrollo compartidos entre universidades públicas y privadas. Un aspecto significativo de su producción científica está determinado por la centralidad del conocimiento, más a nivel de las universidades de la capital que de provincias. También se evidencia la priorización de ciertos dominios investigativos, sobre todo, de lo cognitivo sobre lo procedimental y actitudinal, de lo individual sobre lo colectivo, y de los contenidos disciplinares sobre los transversales. A nivel de los diseños metodológicos, los estudios analizados revelan preferencias, de diseños experimentales y correlacionales sobre los descriptivos, así como la recurrencia a una variedad de sujetos de estudio, no solo de estudiantes, sino, también, de directivos y docentes. Resalta, asimismo, el predominio de los cuestionarios basados en la escala de Likert para la recolección de la información, y, básicamente, elaborado por los autores y autoras; así como un énfasis en pruebas paramétricas para la comprobación de las hipótesis.

Palabras Clave: Blended Learning, Aprendizaje semipresencial, Tendencias metodológicas, Campos de conocimiento, Tesis universitarias.

\section{Abstract:}

Blended learning, which has become a normalized mode of teaching, entails new links with educational surroundings and subjects, becoming, in itself, a topic of research and knowledge. This study looks at forty-five undergraduate and graduate student thesis abstracts by students who graduated from universities in Peru and which are archived at the Peruvian Registry for Research Papers (RENAT). Results purport to more thesis executed at the graduate level, particularly in the past five years. There is also evidence 
of shared research interests between public and private universities. Research on the scientific attributes of BL and the focus on knowledge is more prevalent at universities in Lima, the capital, rather than in provincial areas. In addition, certain research areas were prioritized, such as cognitive studies over procedural and attitudinal; the individual versus the collective whole and disciplinary subject versus cross-cutting. The methodology design, the analyzed theses reveal a penchant towards experimental and correlational designs for descriptive studies on a broad variety of study subjects. This was prevalent not only for students but for school administrators and teachers with a predominance of Likert scale - based questionnaires for data collection prepared by the authors themselves with an emphasis on parametric tests for hypothesis testing.

KEYWORDs: Blended Learning, Methodological Trends, Knowledge Fields, University Thesis Reports.

\section{INTRODUCCIÓN}

La emergencia de las Tecnologías de la Información y de la Comunicación (TIC) ha supuesto cambios y renovaciones en los procesos educativos, al adaptarlos a las necesidades y demandas de los usuarios (Bonk, 2009). En esa orientación, la modalidad Blended Learning (BL) se instituye como una formación normalizada, que va más allá de la sola combinación de espacios formativos (presencialidad y virtualidad), tendiendo a la integración de las estrategias educativas (Aguaded y Cabero, 2013; Turpo y Hernández, 2014) y a una continuidad envolvente de procesos y recursos formativos (Marguliex, McCracker y Catrambone, 2016; Osguthorpe y Graham, 2003). Propiamente, el BL avanza hacia una presencia liberada de la dicotomía presencial y en línea (García, 2005), y a una inevitable convergencia pedagógica y tecnológica (García, 2018).

El BL está posibilitando un mayor acceso a la educación, dada su incorporación e implementación, incrementando, de ese modo, las oportunidades para aprender más profunda y significativamente (Porter, Graham, Spring, y Welch, 2014; Garrison y Kanuka, 2004). La modalidad contribuye intensamente a una mayor interactividad a partir de una profunda implicación tutorial, así como de una intensiva gestión de la tecnología o la capacidad de agencia, entre otros factores (Sembiring, 2018). En conjunto, dichos componentes expresan el potencial de logros efectivos como estrategia institucional (Porter, Graham, Bodily, y Sandberg, 2016; Kenney, y Newcombe, 2011), básicamente, un incremento sustancial en la comprensión, interacción y participación de los agentes y sujetos educativos.

El BL integra las experiencias del aprendizaje convencional o face to face con el aprendizaje en línea o $e$ learning (Bonk y Graham, 2012; Turpo, 2010; Turpo, 2015). La modalidad cambia las formas de aprender y/o construir conocimientos, dada la disolución de las fronteras espacio-temporales y la maleabilidad de los canales de formación, generando una conveniente rentabilidad pedagógica (Graham, 2006; Reynard, 2007). Esencialmente, según Centeno, González, López y Mateos (2016) y Olejarczuk (2014), el BL activa los flujos comunicacionales, revitalizando los vínculos significativos de la colaboración mediada por la tecnología, representando una incitante posibilidad para alcanzar las competencias que consientan superar progresivamente los desafíos sociales.

En la actualidad, el BL en Perú configura un espacio concreto de conocimiento (Turpo y Hurtado, 2019; Turpo y García, 2019), cuyo develamiento implica un estudio de sus campos de intervención, así como de las tendencias metodológicas subyacentes. La exploración metódica de su evolución involucra registrar sus progresos y ajustes, los factores relevantes, etc., reconociendo las configuraciones dominantes, y crecimiento y proyecciones. De esa manera, se explicitan los desafíos de la productividad científica, consintiendo la comprensión de sus matices característicos (Siemens, Gašević, y Dawson, 2015). Abordar la investigación comprende revelar los efectos del entorno en su determinación, así como hacer evidente las tendencias (De Filippo y Fernández, 2002), los intereses investigativos, los enfoques y los espacios de oportunidad y hallazgos más destacables (Duarte, Guzmán y Yot, 2018).

El análisis de la producción científica sobre el BL en las universidades peruanas resulta de interés, dada la ausencia de investigaciones que den cuenta del estado de la cuestión. En esa intención, se trata de mostrar sus avances, dificultades y perspectivas. Asimismo, identificar líneas temáticas de investigación 
para la continuidad y sostenibilidad de futuros estudios. De ese modo, se podrá determinar escenarios y condiciones más plausibles para el intercambio formativo entre sujetos y agentes involucrados en esta modalidad. Contar con una revisión actualizada de la literatura sobre el devenir del BL, contribuirá a evaluar y considerar, pertinente y oportunamente, intervenciones tecno-pedagógicas fundadas en evidencias científicas y apropiadas a la realidad nacional. En concreto, el estudio proveerá información sobre los campos de conocimiento y las orientaciones metodológicas predominantes en las investigaciones universitarias sobre el BL, acercándonos a su comprensión.

\section{MÉTodo}

El acercamiento a las experiencias concretadas por las investigaciones implica recurrir a la revisión sistemática de las tesis universitarias sobre la modalidad BL sustentadas en las universidades peruanas. Un proceso que va más allá de determinar solo la productividad, sino que involucra también un análisis de su desempeño como campo de conocimiento dentro de una comunidad académica (Oyarzun, 2008) y de su devenir como campo de investigación. Siguiendo a Bourdieu (1987), se trata de reconocer las relaciones que definen su producción científica, propiamente, los posicionamientos del poder académico y de investigación a partir de reglas o tendencias que legitiman su conformación en el tiempo y los contextos en los cuales se desarrolla.

El reconocimiento y análisis de la producción científica sobre el BL se inicia con la definición de los criterios de inclusión para la recuperación de las tesis universitarias del Repositorio Nacional de Trabajos de Investigación (RENATI), siendo estos:

- Términos de búsqueda de información: Blended Learning y semipresencial.

- Temporalidad de los informes de tesis (de pregrado y postgrado): 2008-2019 (marzo).

- Acceso no restringido a los informes de tesis.

- Informes de tesis de naturaleza empírica.

Se consideró como criterios de exclusión:

- Informes de planes de mejora/negocio/innovación/ensayos (no tesis).

La secuencia de análisis que llevó a la determinación de la muestra de estudio que se incluye en la Figura 1. 


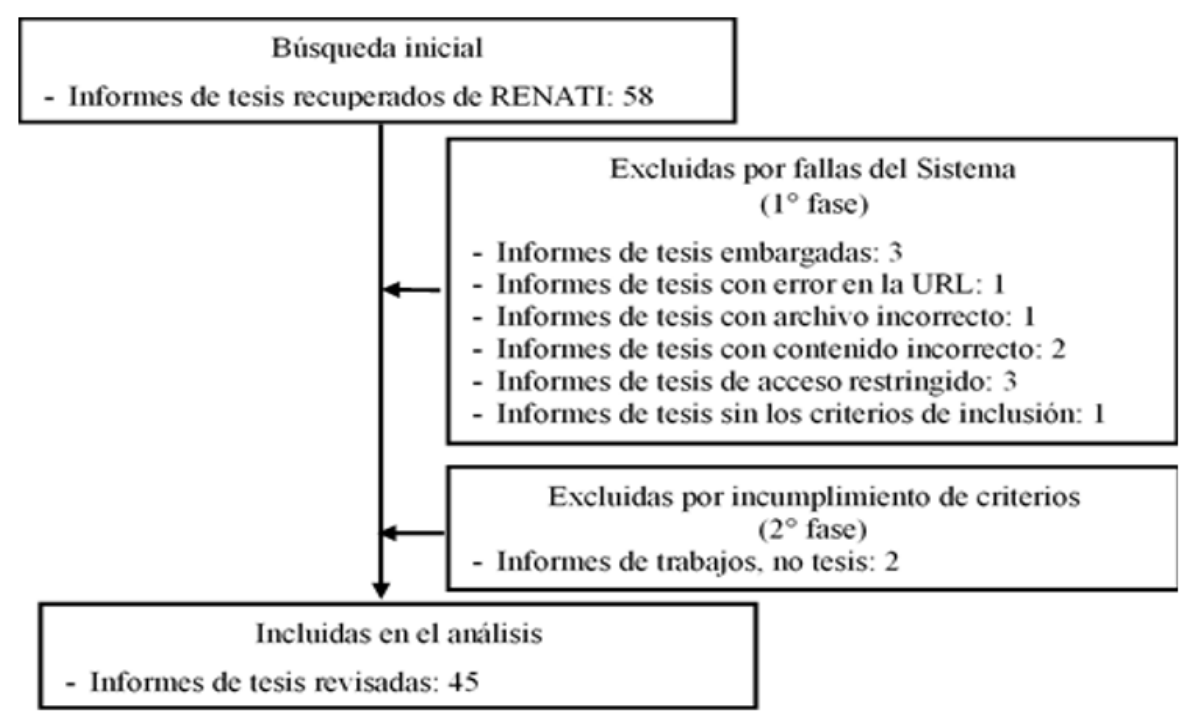

FIGURA 1

Secuencia de determinación de la muestra de estudio Fuente: elaboración propia

Finalmente, fueron 45 los informes de tesis seleccionados, y posteriormente analizados.

\section{Resultados}

El análisis elaborado a partir de las tesis seleccionadas (Tabla 1) se representa en la Figura 2, desde la comparación y contraste de los datos ordenados (Okoli y Schabram, 2010), evidenciando los campos de conocimiento y las tendencias metodológicas.

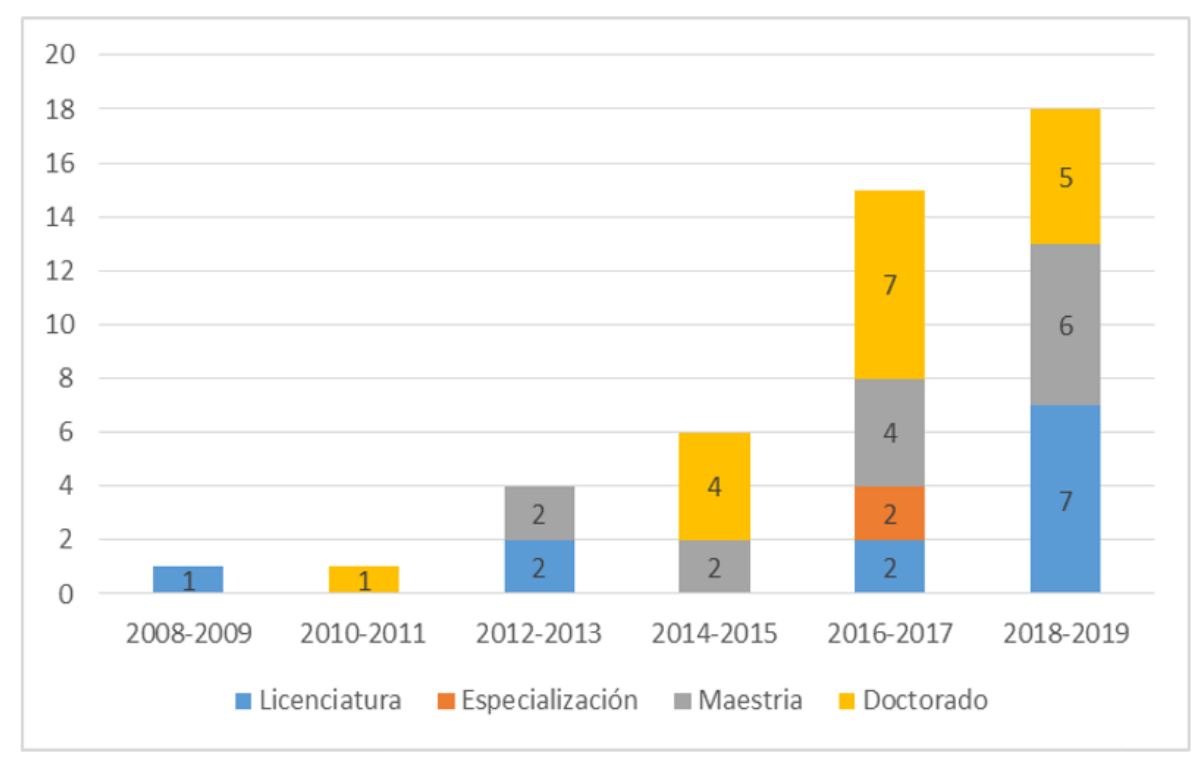

FIGURA 2

Evolución de la producción científica en el BL en Perú (2008-2019)

Fuente: elaboración propia

La producción científica de las tesis sobre el BL se concentra en los estudios de posgrado (Maestría y Doctorado): 31 de las 45 (69\%) lo evidencian. Los dos últimos bienios (2016-2019) patentizan su creciente 
interés investigativo, señalando su "impacto positivo y ajustabilidad al entorno donde se desarrolle [...], resaltándola como eficaz y productiva” (González, Perdomo, y Pascuas, 2017, p. 151).

\section{CAMPOS DE CONOCIMIENTO SOBRE EL BL ABORDADOS EN LAS TESIS UNIVERSITARIAS}

Los énfasis investigativos de los estudios sobre el BL exhiben las concentraciones o agrupaciones de su campo de conocimiento y de sus aplicaciones en el ámbito educativo.

TABLA 1

Evolución temporal de los campos de conocimiento sobre el BL

\begin{tabular}{|c|c|c|c|c|c|c|}
\hline $\begin{array}{l}\text { Campos de } \\
\text { conocimiento }\end{array}$ & $\begin{array}{l}\text { Sub-campos } \\
\text { de } \\
\text { conocimiento }\end{array}$ & $2008-2011$ & $2010-2011$ & $2012-2015$ & 2016-2019 & Total \\
\hline \multirow{12}{*}{$\begin{array}{l}\text { Docencia } \\
\text { universitaria }\end{array}$} & $\begin{array}{l}\text { Desarrollo de } \\
\text { capacidades }\end{array}$ & & 1 & 2 & & 3 \\
\hline & $\begin{array}{l}\text { Desempeño } \\
\text { docente }\end{array}$ & & & 2 & 3 & 5 \\
\hline & $\begin{array}{l}\text { Enseñanza de } \\
\text { la }\end{array}$ & 1 & & 1 & & 4 \\
\hline & comunicación & & & & 2 & \\
\hline & $\begin{array}{l}\text { Enseñanza de } \\
\text { la }\end{array}$ & & & & & 4 \\
\hline & estomatología & & & 2 & 2 & \\
\hline & $\begin{array}{l}\text { Enseñanza de } \\
\text { la física }\end{array}$ & & & & 1 & 1 \\
\hline & $\begin{array}{l}\text { Enseñanza de } \\
\text { la tecnología }\end{array}$ & & & 1 & 2 & 3 \\
\hline & $\begin{array}{l}\text { Enseñanza de } \\
\text { la matemática }\end{array}$ & & & 1 & & 1 \\
\hline & $\begin{array}{l}\text { Enseñanza del } \\
\text { inglés }\end{array}$ & & & 1 & 3 & 4 \\
\hline & $\begin{array}{l}\text { Formación } \\
\text { personal }\end{array}$ & & & & 2 & 2 \\
\hline & $\begin{array}{l}\text { Gestión del } \\
\text { aprendizaje }\end{array}$ & & 1 & & 2 & 3 \\
\hline \multirow{5}{*}{$\begin{array}{l}\text { Educación } \\
\text { básica }\end{array}$} & $\begin{array}{l}\text { Calidad } \\
\text { educativa }\end{array}$ & & & & 1 & 1 \\
\hline & $\begin{array}{l}\text { Desempeño } \\
\text { docente }\end{array}$ & & & & 2 & 2 \\
\hline & $\begin{array}{l}\text { Enseñanza de } \\
\text { la }\end{array}$ & & & 1 & 1 & 2 \\
\hline & comunicación & & & & & \\
\hline & $\begin{array}{l}\text { Enseñanza del } \\
\text { inglés }\end{array}$ & & & & 1 & 1 \\
\hline \multirow{2}{*}{$\begin{array}{l}\text { Educación } \\
\text { tecnológica }\end{array}$} & $\begin{array}{l}\text { Enseñanza de } \\
\text { la matemática }\end{array}$ & & & 1 & 1 & 2 \\
\hline & $\begin{array}{l}\text { Desempeño } \\
\text { docente }\end{array}$ & & & & 1 & 1 \\
\hline \multirow{4}{*}{$\begin{array}{l}\text { Formación } \\
\text { continua }\end{array}$} & $\begin{array}{l}\text { Gestión del } \\
\text { aprendizaje }\end{array}$ & & & & 1 & 1 \\
\hline & $\begin{array}{l}\text { Desempeño } \\
\text { docente }\end{array}$ & & & 3 & 1 & 4 \\
\hline & Teletrabajo & & & & 1 & 1 \\
\hline & Total & 1 & 2 & 15 & 27 & 45 \\
\hline
\end{tabular}

Fuente: elaboración propia

La producción científica sobre el BL se agrupa alrededor de cuatro campos o áreas de conocimiento, definidos alrededor de los niveles formativos (Tabla 1). Los subcampos que los conforman expresan particularidades que resaltan su evolución. Del total de investigaciones universitarias, el 67\% (30 de 45) gira 
en torno a la docencia universitaria. En menor término se encuentran, como objeto de estudio predominante o campo hegemónico de conocimiento, el resto de niveles formativos. El énfasis asignado a las experiencias universitarias muestra la orientación de los sujetos que investigan sobre el BL, así como hacen explícita sus tendencias de desarrollo (Turpo y Hurtado, 2019).

Los estudios sobre el BL en las universidades peruanas grafican los campos de intervención educativa, básicamente, del énfasis formativo en contenidos disciplinares (orientados a una sola disciplina académica) y contenidos transversales (orientados al entrecruce entre varias disciplinas), como se aprecia en la Tabla 2.

TABLA 2

Orientación temática en los campos de conocimiento sobre el BL

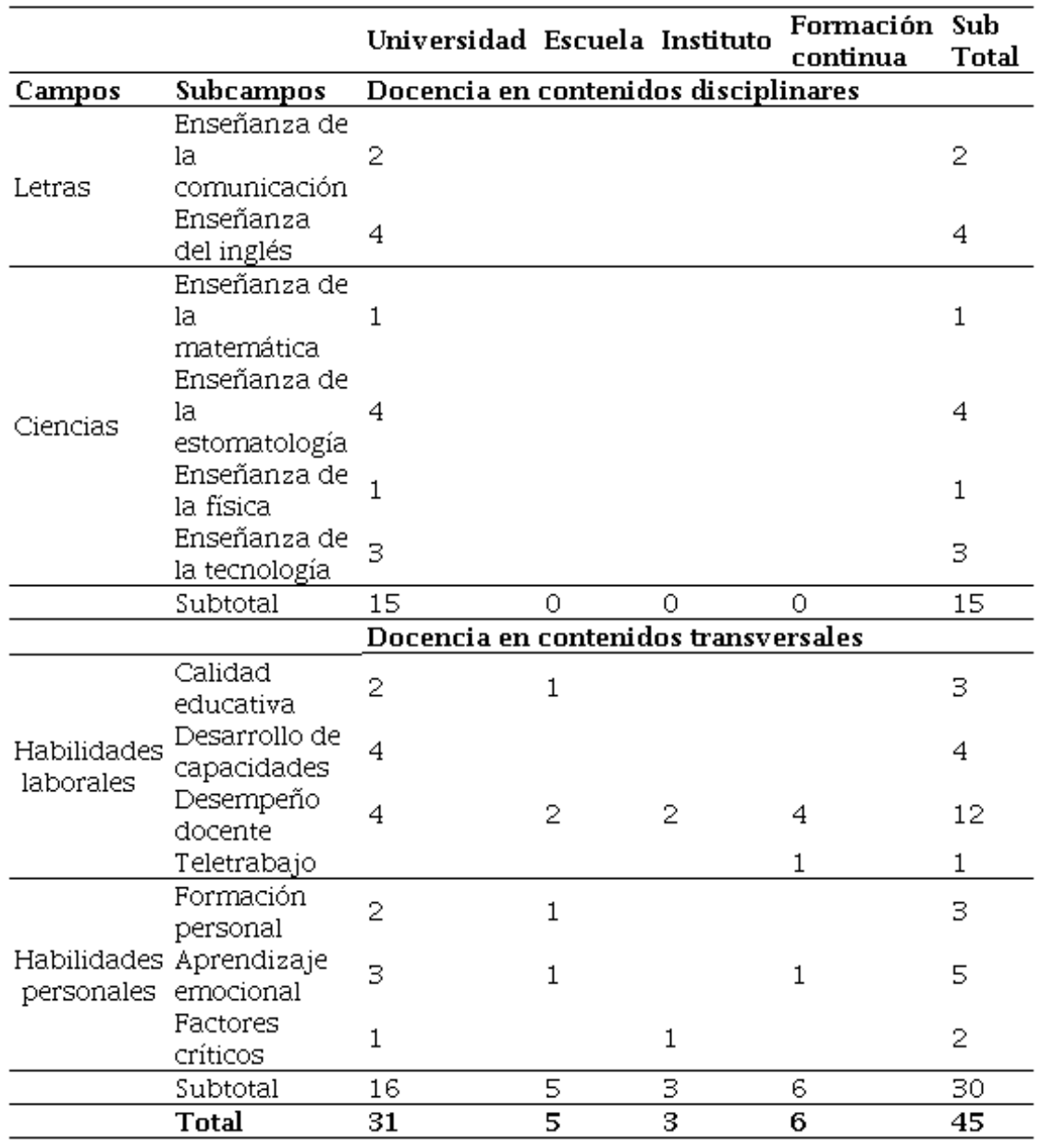

Fuente: elaboración propia

Los estudios sobre la docencia de determinados contenidos transversales convoca un mayor interés investigativo, 67\% (30 de 45), situándose como campo de conocimiento preponderante para la formación universitaria. En la enseñanza de los contenidos disciplinares sobresale el énfasis en la docencia de asignaturas de ciencias ( 9 de 15). Esta diversidad distributiva de campos y subcampos expresa las múltiples posibilidades de aplicación del BL como modalidad formativa, por cuanto, no excluye campos de saber, contrariamente, asume la variedad de manifestaciones de conocimiento, y de su distribución en los diferentes niveles formativos (Heinze, y Procter, 2004; Turpo, 2010).

Las intervenciones didácticas abordadas en los estudios sobre el BL buscan fortalecer habilidades que trascienden el dominio disciplinar, a fin de asegurar una mayor comprensión y, por ende, el desarrollo de 
habilidades integradas (Sánchez, 2012) para la formación profesional y sus repercusiones a nivel práctico (Duarte, et al., 2018). De ese modo, se hace evidente su presencia social e institucional.

\section{Tendencias Metodológicas En LAS INVESTIGACIONES SOBRE EL BL}

En las tesis analizadas se encontró una diversidad de términos que remiten a las metodologías abordadas (Metodología, Marco metodológico, Diseño metodológico, Metodología de la investigación, Método, Medios y materiales, Método, Técnica e Instrumentos y Marco operativo de la investigación) en el estudio del BL. Tales denominaciones conforman los capítulos II, III o IV de los informes; y en pocos casos, de los anexos de las tesis revisadas. Sobre la base de tales denominaciones se reconocen los aspectos metodológicos desde los componentes o elementos constitutivos. Como muestra la Tabla 3, la orientación investigativa dominante en los estudios sobre el BL en las tesis universitarias está en el enfoque cuantitativo, 82\% (37 de 45). Este enfoque es ampliamente considerado en los informes de postgrado (31 de 45). El resto de los enfoques de investigación es subvaluado o menospreciado como tendencia metodológica.

TABLA 3

Enfoques de investigación en las tesis sobre el BL en las universidades peruanas

\begin{tabular}{|c|c|c|c|c|}
\hline \multirow{2}{*}{ Titulación } & \multicolumn{3}{|c|}{ Enfoque de investigación } & \multirow{2}{*}{-Tota } \\
\hline & Cuantitativo & Cualitativo & Mixto & \\
\hline Doctorado & 15 & 1 & 1 & 17 \\
\hline Maestría & 13 & 1 & & 14 \\
\hline Especialización & 2 & & & 2 \\
\hline Licenciatura & 7 & 5 & & 12 \\
\hline Total & 37 & 7 & 1 & 45 \\
\hline
\end{tabular}

Fuente: elaboración propia

La preponderancia del enfoque cuantitativo, revelada en la Tabla 3, indica la presencia de diseños basados en la comprobación de los resultados de la investigación mediante mediciones estadísticas, un aspecto revelador de los énfasis investigativos predominantes en las universidades peruanas (Turpo y García, 2019). La tendencia del enfoque cuantitativo en las investigaciones de las universidades peruanas es revelada también en un estudio reciente de Diaz y Sime (2019), al analizar los estudios de doctorado en educación en Perú.

TABLA 4

Diseños de investigación de las tesis sobre el BL en las universidades peruanas

\begin{tabular}{lllllll}
\cline { 2 - 5 } $\begin{array}{l}\text { Titulación obtenida } \\
\text { Diseños de } \\
\text { investigación }\end{array}$ & Doctorado & Maestría & Especialización & Licenciatura & Total \\
\hline Correlacional & 5 & 6 & 1 & 2 & 14 \\
Cuasiexperimental & 8 & 3 & & 2 & 13 \\
Descriptivo & 3 & 3 & & 4 & 10 \\
Explicativo & 1 & 1 & & 3 & 5 \\
Pre-experimental & 1 & 1 & 1 & $\mathbf{1 1}$ & $\mathbf{4 5}$ \\
\hline Total & $\mathbf{1 8}$ & $\mathbf{1 4}$ & $\mathbf{2}$ &
\end{tabular}

Fuente: elaboración propia

Los diseños de investigación que priman (véase la Tabla 4) responden a estudios que implican la manipulación de variables (cuasi y pre-experimentales): 16 de 45 (36\%) se inscriben en la dinámica de provocación del fenómeno. Un proceso instituido desde el BL, como variable independiente, cuyos efectos 
se miden en los resultados, a través de la comparación. Para Campbell y Stanley (1967), permiten así, "verificar los cambios educacionales y el único modo de establecer una tradición acumulativa en la cual puedan introducirse cambios sin peligro de que ocurra un caprichoso descarte de la antigua sabiduría a favor de novedades inferiores" (p. 172). Los diseños que siguen en orden de priorización están dados por los diseños correlacionales (31\%), descriptivos (22\%), y explicativos (11\%). En términos generales, los diseños cuantitativos asumen la prueba de hipótesis estadística para la comprobación de sus efectos.

TABLA 5

Los sujetos de estudio en las tesis sobre el BL en las universidades peruanas

\begin{tabular}{|c|c|c|c|c|c|}
\hline \multirow{2}{*}{$\begin{array}{l}\text { Sujetos de } \\
\text { estudio }\end{array}$} & \multicolumn{4}{|c|}{ Titulación obtenida } & \multirow{2}{*}{ Total } \\
\hline & Doctorado & Maestría & Especialización & Licenciatura & \\
\hline $\begin{array}{l}\text { Directivos de } \\
\text { IIEE }\end{array}$ & 2 & 1 & & 1 & 4 \\
\hline $\begin{array}{l}\text { Docentes de } \\
\text { educación } \\
\text { básica. }\end{array}$ & 1 & 1 & & 2 & 4 \\
\hline $\begin{array}{l}\text { Docentes } \\
\text { universitarios }\end{array}$ & 1 & & & & 1 \\
\hline $\begin{array}{l}\text { Estudiantes con } \\
\text { NEE }\end{array}$ & & & 1 & & 1 \\
\hline $\begin{array}{l}\text { Estudiantes de } \\
\text { tecnológicos }\end{array}$ & 1 & 1 & & & 2 \\
\hline $\begin{array}{l}\text { Estudiantes de } \\
\text { secundaria }\end{array}$ & 3 & & & 2 & 5 \\
\hline $\begin{array}{l}\text { Textos } \\
\text { autoinstructivos }\end{array}$ & & 1 & & & 1 \\
\hline $\begin{array}{l}\text { Universitarios } \\
\text { de posgrado }\end{array}$ & 3 & & & 2 & 5 \\
\hline $\begin{array}{l}\text { Universitarios } \\
\text { de pregrado }\end{array}$ & 6 & 10 & 1 & 1 & 18 \\
\hline Total & 18 & 14 & 2 & 11 & 45 \\
\hline
\end{tabular}

Fuente: elaboración propia

En los abordajes metodológicos de las investigaciones sobre el BL en las universidades peruanas, como se observa en la Tabla 5, participa una diversidad de sujetos como parte del estudio. El grupo de sujetos predominante son estudiantes universitarios, fundamentalmente de pregrado, que participan en 18 de las 45 investigaciones (40\%). Otro sector de sujetos de estudio está dado por estudiantes de secundaria, así como de directivos y docentes de educación básica. En esencia, se considera tan solo a sujetos de la educación formal o escolarizada, mas no a los de educación no formal o alternativa. Este último grupo de sujetos es el de mayor atención de otros contextos formativos, dada las características de los procesos sociales y educativos en los que participan (Porter et al., 2014).

En cuanto a las técnicas e instrumentos de recolección de información, las figuras siguientes (Figura 3 y Figura 4) resumen las preferencias consideradas en las investigaciones sobre el BL. 


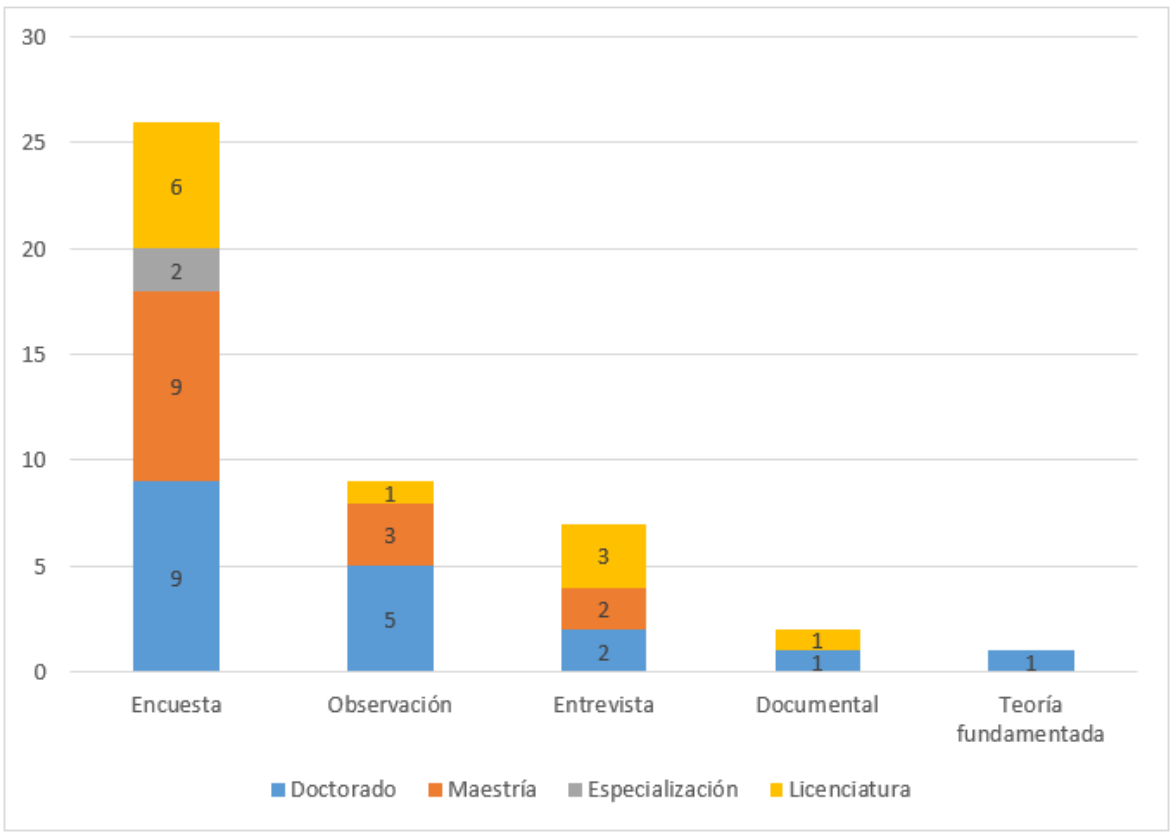

FIGURA 3

Técnicas de recolección de información preferidas en las tesis sobre BL

Fuente: elaboración propia

La técnica predominante en la recolección de la información en las investigaciones sobre el BL es la encuesta, utilizada en 26 de 45 estudios (58\%), prioritariamente, en tesis de postgrado (maestría y doctorado). La priorización de las encuestas evidencia el énfasis de las investigaciones basadas en enfoques cuantitativos (Turpo y García, 2019). Son preferidas también, la observación y la entrevista, y en menor grado, las técnicas relacionadas con las investigaciones cualitativas.

En torno a los instrumentos de recolección de datos, se presentan propensiones a su uso, básicamente, en razón a las técnicas priorizadas (véase la Figura 4). 


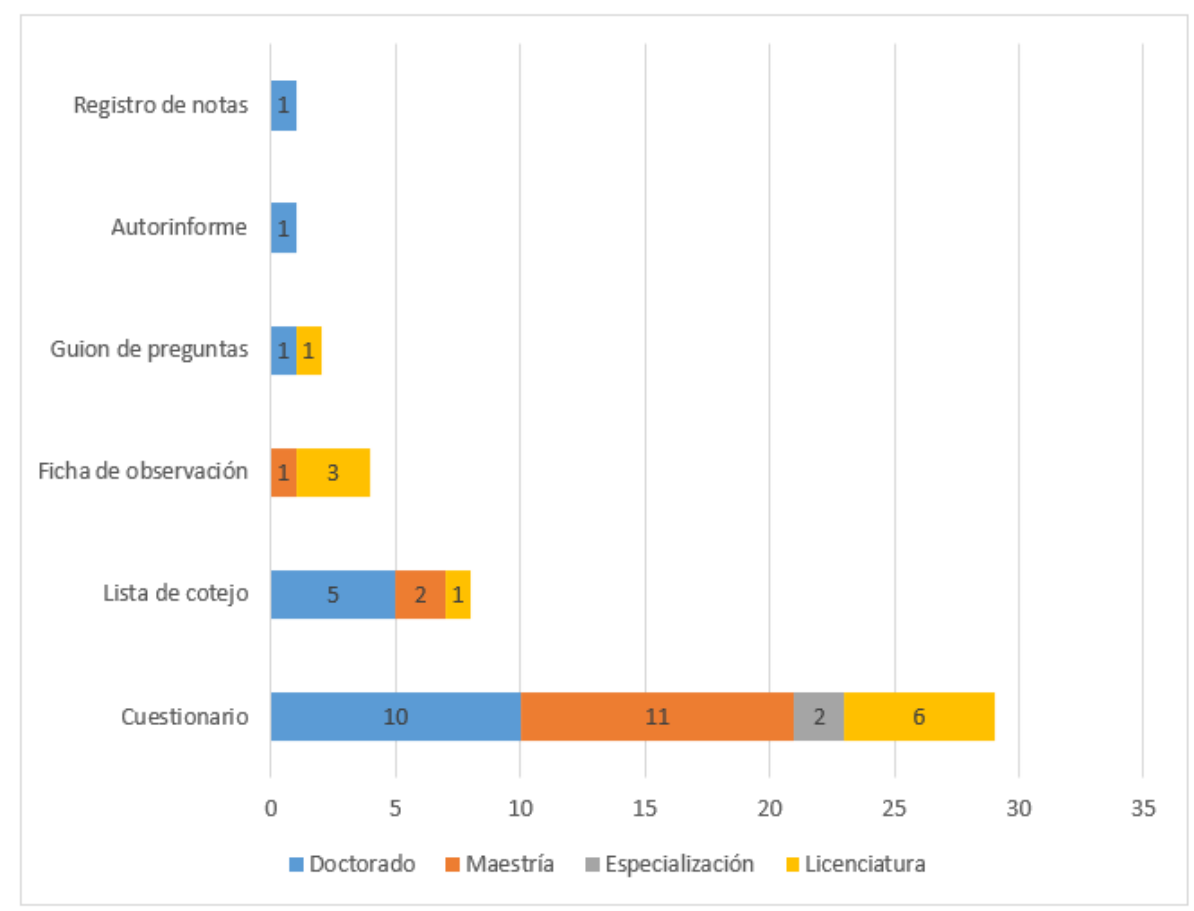

FIGURA 4.

Instrumentos de recolección de datos preferidos en las tesis sobre BL Fuente: elaboración propia

Por la prevalencia de los diseños cuantitativos en las investigaciones sobre el $\mathrm{BL}$, la encuesta se constituye en la técnica de recolección de información de uso prioritario. La utilización de la encuesta permite la obtención de información, de modo económico, y en grandes volúmenes, facilitando el procesamiento e interpretaciones, de forma más rápida y eficaz. Para García (1993), la encuesta permite contar con una muestra representativa de la población, y a partir de ahí, explicar una serie de características. El instrumento priorizado en las encuestas es el cuestionario, dado que responde plenamente a dichas intenciones: 28 de 45 investigaciones (62\%) expresan su uso. El resto de instrumentos son menos utilizados, evidenciando la escasa importancia que se asigna a los otros diseños de investigación.

\section{Conclusiones}

El análisis de las orientaciones teóricas y las tendencias metodológicas sobre el BL de las tesis universitarias sustentadas en Perú, han permitido su identificación como campo de estudio emergente, posibilitando el logro de los objetivos del estudio. Los resultados evidencian las áreas de conocimiento abordadas, así como las orientaciones metodológicas enfatizadas. Las ausencias y hegemonías patentizadas resumen la evolución de la modalidad, tanto temporal como formativa, las particularidades temáticas que la definen, así como, los enfoques y diseños de investigación, los sujetos y técnicas e instrumentos que los investigadores universitarios privilegian.

El estudio sobre el BL evidencia las concentraciones de estudios producidos en determinados contextos, así como su evolución temporal. Sobre esto último, se aprecia en los últimos años un creciente interés investigativo en las universidades peruanas, más a nivel de investigaciones de postgrado que en el de pregrado. Esta diferenciación revela no solo la adopción y normalización de la modalidad, sino también de su reconocimiento como una oportunidad para seguir formándose (Porter et al., 2014; Garrison y Kanuka, 2004). Desde esa perspectiva, el BL guarda consonancia con las demandas sociales y educativas, 
aunque presenta un uso distante en aspectos tecnológicos de otras latitudes; más se avanza a una progresiva aproximación.

La identificación de los campos de conocimientos del BL desvelados por las investigaciones universitarias muestra los énfasis de uso, básicamente, para la formación reglada, y prioritariamente, de carácter universitario, y en campos disciplinares y transversales, tratados con amplia significatividad. De ese modo, resaltan la diversidad de contextos formativos, aunque cada escenario evidencia la peculiaridad de los matices que marcan su decurso (Siemens et al., 2015). Las experiencias analizadas se centran especialmente en la formación autónoma para la inserción en el mundo profesional (Centeno et al., 2016; Olejarczuk, 2014) a partir del empleo de soportes tecnológicos que potencian la enseñanza y minimizan los problemas del aprendizaje.

Entre las orientaciones metodológicas registradas en las investigaciones sobre el BL, se observa la prevalencia de determinadas tendencias en los enfoques investigativos, más de carácter cuantitativo que cualitativo, así como de diseños de investigación, que presuponen la manipulación de variables (cuasiexperimental, con grupo de control y pre-experimental, sin grupo de control), seguido de diseños correlacionales y descriptivos. Tales énfasis distan de lo encontrado por Islas (2014), de una priorización de estudios documentales; mas sí se coincide en el limitado uso de los enfoques cualitativos y mixtos. Resalta también la recurrencia en las investigaciones a una variedad de sujetos de estudio, retratando las múltiples posibilidades formativas del BL, así como de las renovadas formas de interacción didáctica en la gestión de su aprendizaje (Heinze y Procter, 2004; Rebollo, García, Barragán, Buzón y Vega, 2008).

Los hallazgos de la investigación exponen los logros alcanzados y las limitaciones al priorizar ciertos dominios, que para Johnson y Marsh (2014), expresan un sentido de individualización, más que participativo; así como de una mayor preocupación por el desarrollo de competencias académicas que por las habilidades sociales, más centrados en privilegiar los recursos tecnológicos que los apoyos didácticos y tutoriales. Tales tendencias no son las más deseables para la modalidad ni para la formación profesional, menos aún para el desarrollo personal y social. Por el contrario, se requiere de la convergencia de todas las mediaciones (Turpo, 2015; Kim y Bonk, 2006), a fin de afrontar eficazmente las demandas y compromisos de la educabilidad, desde escenarios diversos y complejos, y para procesos cada vez más versátiles.

El recorrido crítico y evolutivo de las investigaciones universitarias sobre el BL en Perú, revela sus concepciones, inscribiéndose como objeto de estudio emergente y de consideración teórica. Expresa, asimismo, su potencial y validez como conocimientos que tributan a los marcos explicativos existentes (García, Aguaded y Bartolomé, 2017). En esencia, el acercamiento a la realidad del BL apertura múltiples posibilidades para futuras investigaciones, entre ellas, las indispensables y sucesivas revisiones de literatura que actualicen su evolución. Además, posibilitará indagar sobre aspectos propiamente formativos, esencialmente, sobre las interacciones didácticas que construyen los sujetos, los recursos virtuales y presenciales empleados en los intercambios académicos y sociales, así como la formación de las y los docentes en procesos tecno-pedagógicos, la satisfacción con los servicios educativos, entre otras. También, cabría indagar sobre aspectos organizativos de la modalidad, tiempos, materiales, diseños instruccionales y demás circunstancias concurrentes.

\section{REFERENCIAS}

Aguaded, I., y Cabero, J. (2013). Tecnologias y medios para la educación en la e-sociedad. Madrid: Alianza.

Bonk, C. (2009). The World is Open: How Web Technology Is Revolutionizing Education. En G. Siemens y C. Fulford (Eds.). Proceedings of ED-MEDIA 2009-World Conference on Educational Multimedia, Hypermedia \& Telecommunications (pp. 3371-3380). Honolulu, USA: Association for the Advancement of Computing in Education (AACE). 
Bonk, C., y Graham, C. (2012). The handbook of blended learning: Global perspectives, local designs. San Francisco, USA: Pfeiffer Publishing.

Bourdieu, P. (1987). What makes a social class? On the theoretical and practical existence of groups. Berkeley journal of sociology, 32, 1-17. Recuperado de https://bit.ly/2wFaKhI

Campbell, D. y Stanley, J. (1967). Diseños experimentales y cuasiexperimentales en la investigación social. Buenos Aires: Amorrortu editores.

Centeno, A., González, M., López, M, y Mateos, P. (2016). Una experiencia de aprendizaje combinado en Estadística para estudiantes de Psicología usando la evaluación como herramienta de aprendizaje. Education in the Knowledge Society, 17(1), 65-85. doi: https://doi.org/10.14201/eks20161716585

De Filippo, D. y Fernández, M. (2002). Bibliometria: importancia de los indicadores bibliométricos. Buenos Aires, Argentina: RICYT.

Diaz, C. y Sime, L. (2019). Las tesis de doctorado en educación en el Perú: Un perfil de la producción académica en el campo educativo. Lima: PUCP.

Duarte, A., Guzmán, M., y Yot, C. (2018). Aportaciones de la formación blended learning al desarrollo profesional docente. RIED. Revista Iberoamericana de Educación a Distancia, 21(1), 155-174. doi: https://doi.org/10.594 4/ried.21.1.19013

García, M. (1993). La encuesta. En: M. García, J. Ibáñez y F. Alvira (eds.). El análisis de la realidad social. Métodos y técnicas de Investigación. (pp. 141-170). Madrid: Alianza Universidad.

García, R., Aguaded, I., y Bartolomé, A. (2017). La revolución del blended learning en la educación a distancia. RIED. Revista Iberoamericana de Educación a Distancia, 21(1), 25-32. doi:https://doi.org/10.5944/ried.21.1.19803

García, L. (2018). Blended learning y la convergencia entre la educación presencial y a distancia. RIED, Revista Iberoamericana de Educación a Distancia, 21(1), 9-22. https://doi.org/10.5944/ried.21.1.19683

García, F. (2005). Estado actual de los sistemas e-learning. Teoría de la Educación. Educación y Cultura en la Sociedad de la Información, 6(2), 1-7. Recuperado de http://campus.usal.es/ teoriaeducacion/rev_numero_06_2/n6_0 2_art_garcia_penalvo

Garrison, D., y Kanuka, H. (2004). Blended learning: Uncovering its transformative potential in higher education. The internet and higher education, 7(2), 95-105. doi: https://doi.org/10.1016/j.iheduc.2004.02.001

González, M., Perdomo, K., y Pascuas, Y. (2017). Aplicación de las TIC en modelos educativos blended learning: una revisión sistemática de literatura. Sophia, 13(1), 144-154. https://doi.org/10.18634/sophiaj.13v.1i.364

Graham, C. (2006). Blended Learning Systems: Definition, Current Trends, and Future Directions. En C. Bonk y C. Graham. (Eds.). The Handbook of Blended Learning: Global Perspectives, Local Designs. (pp. 2-21). San Francisco, USA: Pfeiffer Publishing.

Heinze, A., y Procter, C. (2004). Reflections on the Use of Blended Learning. Education in a Changing Environment. Salford, UK: University of Salford.

Islas, C. (2014). El B-learning: un acercamiento al estado del conocimiento en Iberoamérica, 2003-2013. Apertura, 6(1), 86-97. Recuperado de https://www.redalyc.org/pdf/688/68831999008.pdf

Johnson, C. P. y Marsh, D. (2014). Blended Language Learning: An Effective Solution but Not without Its Challenges. Higher Learning Research Communications, 4(3), 23-41. doi:https://doi.org/10.18870/hlrc.v4i3.213

Kenney, J., y Newcombe, E. (2011). Adopting a blended learning approach: Challenges encountered and lessons learned in an action research study. Journal of Asynchronous Learning Networks, 15(1), 45-57. doi: https://doi .org/10.24059/olj.v15i1.182

Kim, K.J. y Bonk, C.J. (2006). The future of online teaching and learning in higher education. Educause quarterly, 29(4), 22-30. Recuperado de http://faculty.weber.edu/eamsel/Research\%20Groups/On-line\%20Learning/Bo $\mathrm{nk} \% 20(2006) . \mathrm{pdf}$

Marguliex, I., McCracker, W., y Catrambone, R. (2016). A taxonomy to define courses that mix face-to-face and online learning. Educational Research Review, 19, 104-118. doi: https://doi.org/10.1016/j.edurev.2016.07.001 
Okoli, C., y Schabram, K. (2010). A Guide to Conducting a Systematic Literature Review of Information Systems Research. Sprouts. Working Papers on Information Systems, 10(26), 1-56. doi: https://doi.org/10.2139/ssrn.19 54824

Olejarczuk, E. (2014). The e-learning component of a blended learning course. Teaching English with Technology, 14(3), 58-68. Recuperado de https://files.eric.ed.gov/fulltext/EJ1143472.pdf

Osguthorpe, R., y Graham, C. (2003). Blending learning environments: Definitions and directions. The Quarterly Review of Distance Education, 4(3), 227-233. Recuperado de https://www.learntechlib.org/p/97576/

Oyarzun, R. (2008). Ciencia, revistas científicas y el Science Citation Index: o cómo volvernos locos a golpe de números. REDVET. Revista electrónica de Veterinaria, 9(9), 1-5. Recuperado de https://bit.ly/2y71RO9

Porter, W., Graham, C., Spring, K., y Welch, K. (2014). Blended learning in higher education: Institutional adoption and implementation. Computers \& Education, 75, 185-195. doi: https://doi.org/10.1016/j.compedu.2014.02. 011

Porter, W., Graham, R., Bodily, G., y Sandberg, S. (2016). A qualitative analysis of institutional drivers and barriers to blended learning adoption in higher education. The internet and Higher education, 28, 17-27. doi: https://d oi.org/10.1016/j.iheduc.2015.08.003

Rebollo, M., García, R., Barragán, R., Buzón, O., y Vega, L. (2008). Las emociones en el aprendizaje online. RELIEVERevista Electrónica de Investigación y Evaluación Educativa, 14(1). 1 - 23. doi: https://doi.org/10.7203/reliev e.14.1.4201

Reynard, R. (23 de mayo de 2007). Hybrid Learning: Maximizing Student Engagement [Mensaje en blog]. Campus Technology. Recuperado de https://campustechnology.com/articles/2007/05/hybrid-learning-maximizing-stu dent-engagement.aspx

Sánchez, R. (2012). Estrategias didácticas blended learning para la enseñanza de la Geografía Económica en el bachillerato. Revista Educación y Tecnologia, 1, 123-148. Recuperado de http://revistas.umce.cl/index.php/edy tec/article/view/40/pdf

Sembiring, M. (2018). Validating student satisfaction with a blended learning scheme in Universitas Terbuka setting. International Journal of Mobile Learning and Organisation, 12(4), 394-413. doi: https://doi.org/10.1504/ijml o.2018.095166

Siemens, G., Gašević, D., y Dawson, S. (2015). Preparing for the digital university: a review of the history and current state of distance, blended, and online learning. Athabasca University. Recuperado de http://linkresearchlab.org /PreparingDigitalUniversity.pdf

Turpo, O. (2010). Contexto y desarrollo de la modalidad educativa blended learning en el sistema universitario iberoamericano. Revista Mexicana de Investigación Educativa, 15(45), 345-370. Recuperado de http://www.sci elo.org.mx/scielo.php?pid=S1405-66662010000200002\&script=sci_abstract\&tlng=en

Turpo, O. (2015). Perspectiva de la convergencia pedagógica y tecnológica en la modalidad blended learning. Revista De Educación a Distancia, (39), 1-14. Recuperado de https://revistas.um.es/red/article/view/234261

Turpo, O. y García, F. (2019). Orientaciones metodológicas en los estudios sobre el Blended Learning en las universidades peruanas. RISTI - Revista Ibérica en Sistemas y Tecnologias de la Información, (E21), 9-22. Recuperado de https://repositorio.grial.eu/bitstream/grial/1811/1/Turpo01.pdf

Turpo, O., y Hernández, M. (2014). La convergencia pedagógica y tecnológica de la modalidad Blended Learning. En V. Martín-García (Coord.). Blended learning en Educación Superior. Perspectivas de innovación y cambio (pp. 101-119). Madrid: Síntesis.

Turpo, O. y Hurtado, A. (2019). Productividad científica sobre el Blended Learning en el Perú: aproximaciones a su evolución desde las tesis universitarias. Education in the Knowledge Society (EKS), 20, 1-19. doi: https://doi.or g/10.14201/eks2019_20_a19

\section{BY-NC-ND}

